\title{
Quantum nonlinear optics - photon by photon
}

\author{
Darrick E. Chang', Vladan Vuletić ${ }^{1}$ and Mikhail D. Lukin ${ }^{3 \star}$
}

The realization of strong interactions between individual photons is a long-standing goal of both fundamental and technological significance. Scientists have known for over half a century that light fields can interact inside nonlinear optical media, but the nonlinearity of conventional materials is negligible at the light powers associated with individual photons. Nevertheless, remarkable advances in quantum optics have recently culminated in the demonstration of several methods for generating optical nonlinearities at the level of individual photons. Systems exhibiting strong photon-photon interactions enable a number of unique applications, including quantum-by-quantum control of light fields, single-photon switches and transistors, all-optical deterministic quantum logic, and the realization of strongly correlated states of light and matter.

hotons traveling through a vacuum do not interact with each other. This linearity in light propagation, in combination with the high frequency and hence large bandwidth provided by waves at optical frequencies, has made optical signals the preferred method for communicating information over long distances. In contrast, the processing of information requires some form of interaction between signals. In the case of light, such interactions can be enabled by nonlinear optical processes. These processes, which are now found ubiquitously throughout science and technology, include optical modulation and switching, nonlinear spectroscopy and frequency conversion ${ }^{1}$, and have applications across both the physical $^{2}$ and biological ${ }^{3,4}$ sciences.

A long-standing goal in optical science has been the implementation of nonlinear effects at progressively lower light powers or pulse energies. The ultimate limit may be termed 'quantum nonlinear optics' (Box 1) - the regime where individual photons interact so strongly with one another that the propagation of light pulses containing one, two or more photons varies substantially with photon number. Although this domain is difficult to reach owing to the small nonlinear coefficients of bulk optical materials, the potential payoff is significant. The realization of quantum nonlinear optics could improve the performance of classical nonlinear devices, enabling, for example, fast energy-efficient optical transistors that avoid Ohmic heating ${ }^{5}$. Furthermore, nonlinear switches activated by single photons could enable optical quantum information processing and communication ${ }^{6}$, as well as other applications that rely on the generation and manipulation of non-classical light fields ${ }^{7,8}$.

\section{The challenge of making photons interact}

At low optical powers, most optical materials exhibit only linear optical phenomena, such as refraction and absorption, which can be described by a complex index of refraction. However, a sufficiently intense light beam can modify a material's index of refraction, such that the light propagation becomes power-dependent. This is the essence of classical nonlinear optics (Box 1). Large optical fields are required to alter the index of refraction of conventional bulk materials because a strong nonlinear response can only be induced if the electric field of the light beam acting on the electrons is comparable to the field of the nucleus. As a result, early experimental observations of nonlinear optical phenomena, such as frequencydoubling or sum-frequency generation ${ }^{9}$, were achievable only after the development of powerful lasers.
Advances in nonlinear optics over the past four decades have resulted in progressively more efficient nonlinear processes ${ }^{10}$, thus enabling the observation of nonlinear processes at lower and lower light levels. It is natural to inquire if and how these nonlinear interactions can be made so strong that they become important even at the level of individual quanta of radiation. Although this question was addressed in early theoretical studies ${ }^{11-13}$, it has become more pressing with the advent of quantum information science. Specifically, following the pioneering experiments by Turchette and co-workers ${ }^{14}$, much theoretical and experimental effort has been directed towards the realization of two-photon nonlinearities and photonic quantum logic. In the microwave domain, significant progress has been made using either Rydberg atoms in high- $Q$ cavities, or superconducting circuits as 'artificial atoms' (see, for example, the excellent reviews by Haroche and Raimond ${ }^{15}$, and Devoret and Schoelkopf ${ }^{16}$ ). In the optical domain, the probabilistic realization of quantum logic operations using linear optics and photon detection has been actively explored ${ }^{17}$, where the effective nonlinearity in an otherwise linear system arises from the post-selection of photon detection events. Although this approach has recently been used to, for example, implement quantum algorithms in systems of up to four photons ${ }^{18}$, the success rate decreases exponentially with photon number at finite photon detection probabilities, which makes it difficult to scale the process to a larger number of photons or operations. In parallel, researchers have pursued the technologically more challenging - but potentially more powerful and scalable - method of implementing deterministic photon-photon interactions ${ }^{13,19-22}$.

To understand why it is difficult to generate an optical response that is nonlinear at the level of individual photons, let us consider the interaction of a tightly focused laser beam with atoms (Box 1). We would like to determine how many photons it takes to alter the atomic response, which can in turn modify the light propagation. To answer this question, we can think about light propagation in a focused beam as a flow of photons in a cylinder of diameter $d$. The probability of interaction $p$ between one photon and one atom is then given by the ratio of an effective size of the atom as seen by a photon (the atom's absorption cross-section, $\sigma$ ) and the transverse area of the laser beam $\left(\sim d^{2}\right)$. The absorption cross-section is a function of the frequency of light. It reaches its maximum when the light frequency matches the frequency of the atomic transition, with a value of the order of the wavelength of light squared $\left(\sim \lambda^{2}\right)$, giving $p \approx \lambda^{2} / d^{2}$. Because diffraction prevents the focusing of light below the wavelength scale, in free space $d>\lambda$, so typically $p$ « 1 .

${ }^{1}$ Institut de Ciències Fotòniques (ICFO), Mediterranean Technology Park, 08860 Castelldefels, Barcelona, Spain, ${ }^{2}$ Department of Physics and Research Laboratory of Electronics, Massachusetts Institute of Technology, Cambridge, Massachusetts 02139, USA, ${ }^{3}$ Department of Physics, Harvard University, Cambridge, Massachusetts 02138, USA. *e-mail: lukin@physics.harvard.edu 


\section{Box 1 | Physics of photon-photon interactions.}

The interaction between an atom and a photon, confined to a beam of diameter $d$, can be understood from simple geometrical considerations (a). At resonance, the atom has a maximal scattering cross-section that is proportional to the square of the optical wavelength, $\sigma \approx \lambda^{2}$. The probability that a single photon in the beam interacts with the atom is therefore $p \approx \lambda^{2} / d^{2}$, which is typically much smaller than unity. This can be enhanced by using an optical cavity to make a photon interact with an atom multiple times, or by confining light to subwavelength dimensions. The excitation spectrum of a single atom is extremely nonlinear, as the absorption of a single photon saturates the atomic response. This results in strong photon-photon interactions when the atom-photon interaction probability $p$ approaches unity.

The different regimes of nonlinear optical phenomena can be characterized by the interaction strength per photon and the number of photons involved (b). In conventional optical media, the interaction strength per photon is weak, which corresponds to linear optics at a low photon number (light grey box). At a higher photon number, we enter the regime of classical nonlinear optics (dark grey box). Quantum nonlinear optical phenomena occur when the interaction strength per photon becomes large. For a small photon number, strong interactions can be used to achieve quantum control of light fields photon-by-photon (blue box), and to implement photonic quantum gates. A novel regime occurs

Hence a large number of atoms $N \approx 1 / p$ is required to substantially modify the propagation of the light beam. To saturate such an atomic ensemble and thus produce a nonlinear optical response, a correspondingly large number of photons $n \approx N \approx d^{2} / \lambda^{2} \approx 1 / p$ is needed. A number of experiments have attempted to maximize the atom-photon interaction probability $p$ by concentrating laser light to a small area, achieving sizeable atom-photon interaction probabilities of $p \approx 0.05$ with laser beams focused on neutral atoms ${ }^{23,24}$, $p \approx 0.01$ with ions ${ }^{25}$, and $p \approx 0.1$ with molecules on a surface ${ }^{26}$.

In the limit where the atom-photon interaction probability $p$ approaches unity, a single atom can cause substantial attenuation, phase shift or reflection of an incident single photon. At the same time, because a single two-level atom has a highly nonlinear optical response - it cannot absorb or emit more than one photon at a given time - the absorption of a photon drastically changes the atom's response to a second arriving photon. In other words, a pair of simultaneous incident photons will experience an atomic response that is significantly different from the response to a single photon, resulting in an optical nonlinearity at the two-photon level. In the following, we describe several practical methods for reaching the regime of $p \rightarrow 1$ and obtaining strong interactions between individual photons.

\section{Single atoms in cavities}

One technique for enhancing the atom-photon interaction probability beyond what is possible with a tightly focused laser beam is to make the photon pass through the atom repeatedly. This can be achieved by means of an optical cavity ${ }^{27-32}$ (Fig. 1). In this case, the interaction probability is enhanced by the number of bounces the photon makes between the mirrors before leaving the cavity, which is conventionally quantified by the cavity finesse F. By taking the multipass atom-photon interaction into account, we can define a quantity $\eta \approx F \lambda^{2} / d^{2}$ known as the cooperativity ${ }^{32}$; when $\eta » 1$, the interaction probability $p$ approaches unity.

In such a cavity quantum electrodynamics system, the optical nonlinearity arises from the discrete level structure of the atom. In a two-level atom the effect is simply the familiar saturation of atomic

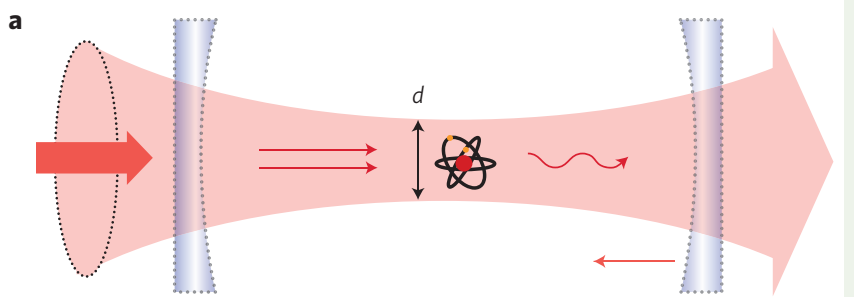

b

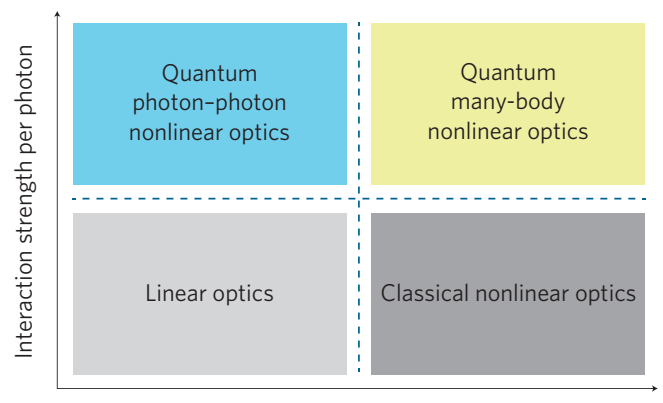

Photon number

when many photons interact simultaneously to produce strongly correlated many-body behaviour (yellow box).

absorption: an atom in the ground state absorbs light, whereas an atom in an excited state emits or amplifies light. A high cooperativity ensures that even a single photon can alter the response of a single atom inside the resonator. In a pioneering early experiment, Turchette and co-workers demonstrated that atomic saturation can be used to shift the phase of one photon by around $\pi / 10$ (ref. 14).

A two-level atom coupled to an optical cavity gives rise to the nonlinear energy level structure of the Jaynes-Cummings model ${ }^{33}$ (Fig. 1). In particular, the strong atom-photon coupling yields an extra interaction energy cost to populate the system with $n$ photons, as compared with an empty cavity in which $n$ photons have an energy corresponding to $n$ times that of a single photon. This feature can be used to generate non-classical light by tuning the excitation laser to the corresponding transition frequency of the nonlinear Jaynes-Cummings ladder, as demonstrated in experiments with a single atom trapped inside a high-finesse optical resonator $27,34,35$ (Fig. 1).

Instead of using real atoms, which must be cooled and trapped inside an optical resonator, it is also possible to use artificial atoms in a solid-state system, such as quantum dots in semiconductors ${ }^{29,36-38}$ or nitrogen-vacancy centres in diamond ${ }^{39,40}$. Artificial atoms typically feature much larger linewidths and hence large optical bandwidths, whereas lithographically fabricated subwavelength-size cavities enable large cooperativities. Such artificial atoms constitute effective two-level systems that have been used to demonstrate a variety of nonlinear effects, including nonlinear phase shifts and optical switching at power levels corresponding to one photon on average ${ }^{29,31}$, and the generation of non-classical light ${ }^{36,37,41,42}$.

Although two-level atoms are capable of generating quantum nonlinearities, they face a number of limitations. Specifically, the short lifetimes associated with electronic excited states prevents atoms from 'remembering' their interaction with photons for long time intervals. This implies that two photons must arrive simultaneously at an atom to interact. In such a case, a two-level atom behaves as a nonlinear frequency mixer, which generates unwanted entanglement between spatial degrees of freedom of photons and thus limits certain processes and applications ${ }^{43,44}$. These limitations can be overcome by employing multilevel atoms with two ground and/or 


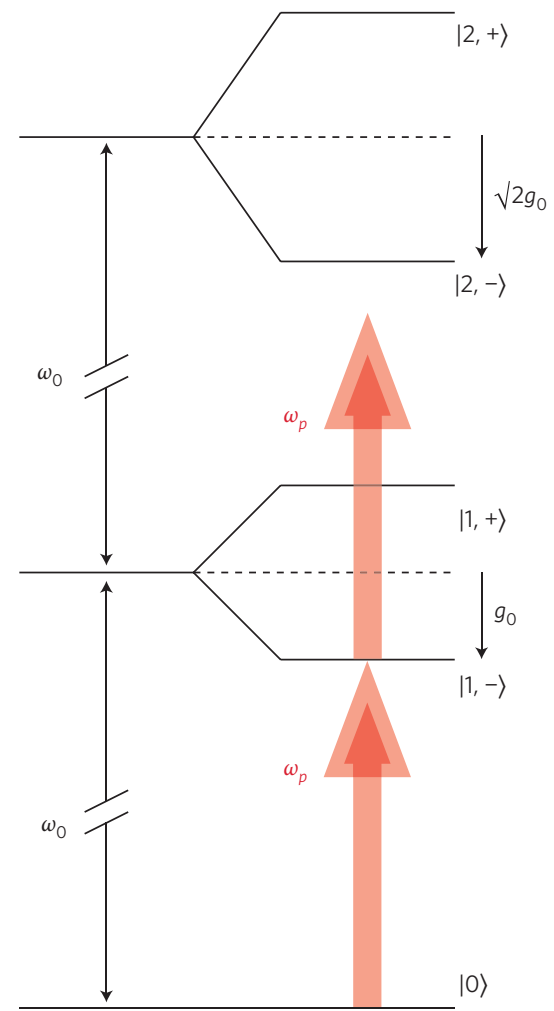

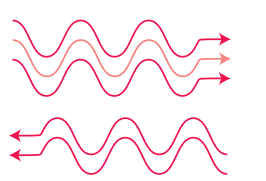

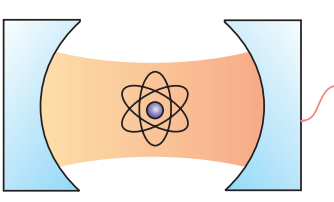

$\mathrm{D}_{1}$
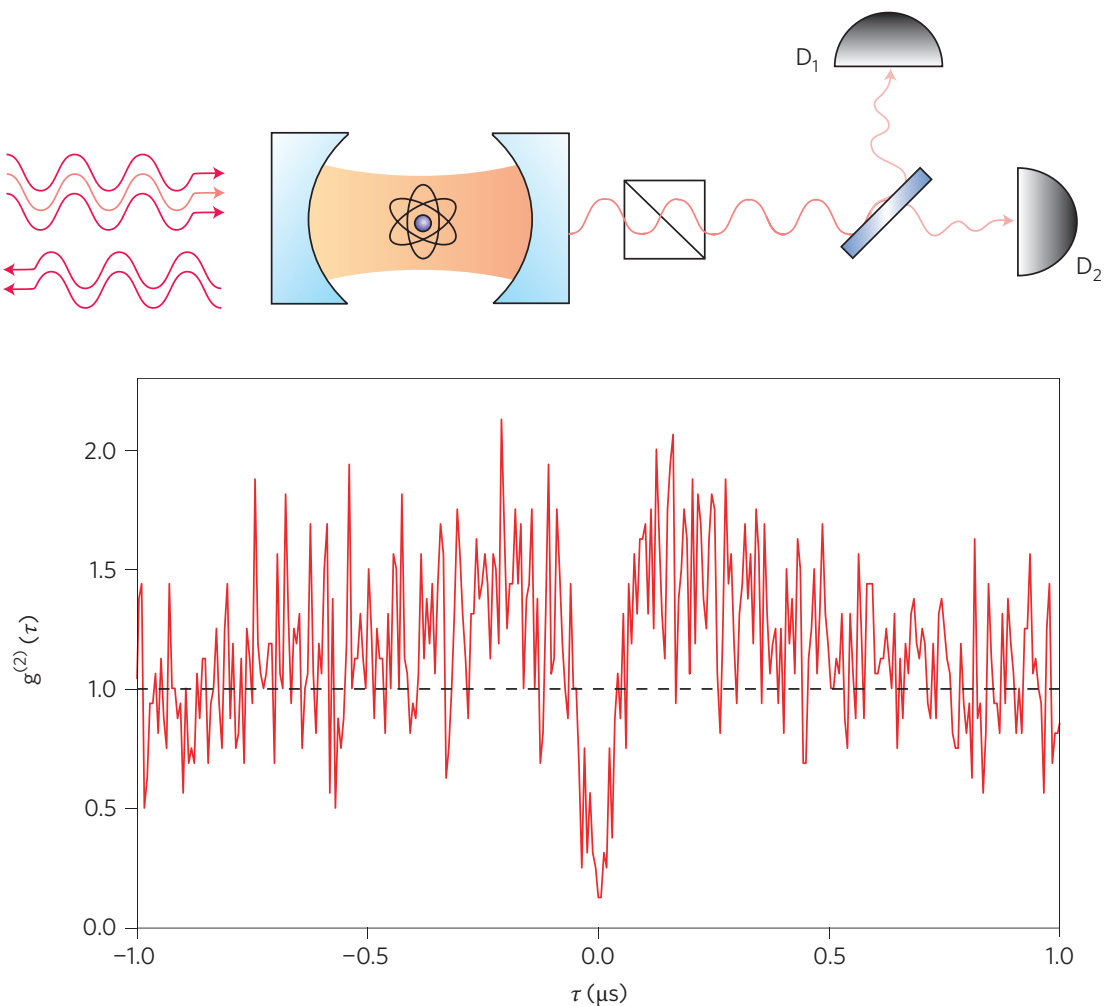

Figure 1 | Quantum nonlinear optics in a cavity. a, The energy spectrum of the Jaynes-Cummings model, which describes a single-mode field coupled to a single atom. Strong interactions result in a mixing of atomic and photonic states, and the energies of the corresponding eigenstates (denoted as $| \pm\rangle)$ are split by an amount proportional to the single-photon Rabi frequency $g_{0}$ and square root of photon number $n$. This dependence results in an anharmonic (nonlinear) energy spectrum. When the system is probed by a laser field of frequency $\omega_{\mathrm{p}}$ tuned to one of the resonances associated with the single-excitation manifold, the system can only transmit one photon at a time, as the doubly excited state is detuned. b, The suppression of two-photon transmission can be observed by splitting the transmitted field and measuring coincident photon detection events at detectors D1 and D2. The probability of detecting a second photon at time $\tau$, given a detection event at $\tau=0$, is given by the second-order correlation function $g^{(2)}(\tau)$. The 'antibunching' dip at $\tau=0, g^{(2)}(0)<1$, is a non-classical signature that reflects the improbable occurrence of two photons being transmitted simultaneously. Figure reproduced from ref. 27, Nature Publishing Group.

metastable states ${ }^{45,46}$, in which quantum coherence can be stored for long periods. Such states enable the implementation of a quantum memory ${ }^{45}$, whereby a quantum state of light can be mapped onto atomic states (Fig. 2). Here, by using an auxiliary laser to implement Raman absorption, an incoming photon can be converted into a long-lived superposition of atomic ground states, as demonstrated by Specht and co-workers ${ }^{47}$ (Fig. 2). Using the first photon to transfer the atom to another ground state switches the transmission or reflection of the atom, thus enabling the first photon to interact with subsequently applied probe photons. Similar approaches have recently allowed researchers to implement a non-destructive photon detector $^{48}$, a quantum phase switch between a single atom and a single photon $^{49}$, and a quantum gate between an atom and a photon ${ }^{50}$. These demonstrations constitute a key enabling technology for quantum networks ${ }^{6}$, where individual, remote quantum bits encoded in atoms are connected and entangled via photonic channels (Fig. 2).

\section{Quantum nonlinear optics using atomic ensembles}

Instead of using a cavity to store a photon in a single atom, it is also possible to map the photon onto the collective states of an atomic ensemble $e^{46,51}$. The central idea is illustrated in Fig. 3a, in which a weak probe field incident on an atomic gas is coupled to a third atomic state $|s\rangle$ by means of a second, stronger laser field the control field. For a weak probe field, the control field induces a spectral transparency window in the otherwise opaque medium through electromagnetically induced transparency ${ }^{46}$ (EIT), and the probe pulse travels at a much reduced speed in the form of a coupled excitation of light and matter. Such a slowly propagating probe light pulse can be manipulated very efficiently. For instance, ramping down the control field reduces the group velocity of the probe pulse to zero, which maps the quantum state of the probe field onto atoms ${ }^{52,53}$. By reversing this procedure, the atomic state can be mapped back onto the probe field propagating in the original direction. If the metastable state $|s\rangle$ is coupled to a fourth state, the system can also be used to produce a strong nonlinear response. A resonant light beam tuned to the $|s\rangle-\left|e^{\prime}\right\rangle$ transition (switch field, shown by the green arrow in Fig. 3a) destroys the quantum interference associated with EIT and blocks transmission of the probe field. Such EIT cross-coupling nonlinearities ${ }^{54}$ and similar effects ${ }^{55}$ at the few-photon level have been observed by means of strong transverse confinement of the light inside optical fibres (Fig. 3b,c).

Recently, these techniques have been extended into the quantum regime by confining either the control or the switching field in a cavity. For example, Tanji-Suzuki and co-workers used an ensemble of three-level atoms to implement vacuum-induced transparency ${ }^{32}-$ an inherently nonlinear system ${ }^{56}$ in which the classical control beam of EIT is replaced by a cavity vacuum field. With four-level atoms, Chen and co-workers have realized an optical transistor gated by just one stored photon ${ }^{57}$.

Quantum nonlinear optics through atom-atom interactions An alternative approach that does not require the use of optical resonators exploits strong atom-atom interactions in the metastable state $|r\rangle$ of an EIT scheme ${ }^{58-61}$ (Fig. 4). If $|r\rangle$ is chosen as a state with 

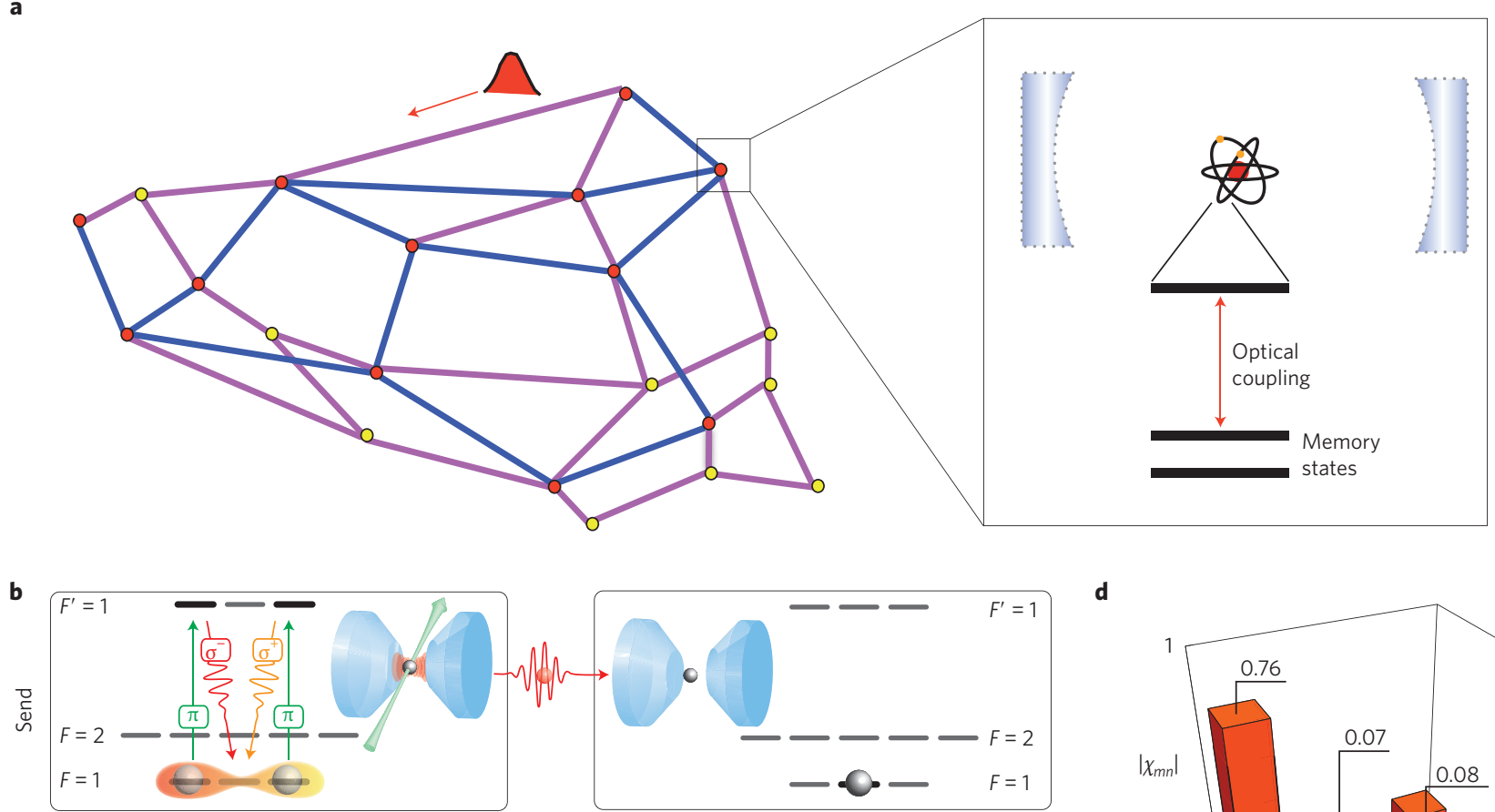

d
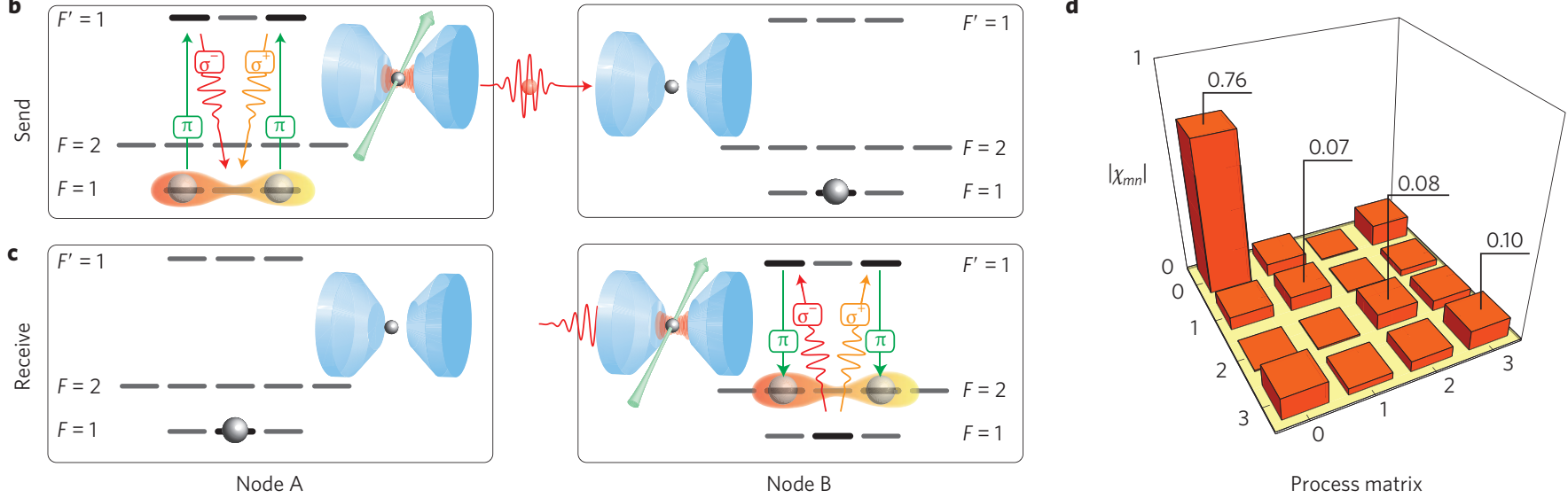

Figure 2 | Quantum networks. a, Schematic of a quantum optical network in which atomic memory nodes can store several quantum bits and process quantum information locally. These nodes are connected via optical fibres that can carry quantum information encoded in photons. Qubits can be mapped from light to atoms mediated, for example, by optical transitions in cavities. b-d, Operation of a two-node quantum network. First, a qubit is stored in a coherent superposition of two Zeeman sublevels of a single atom. It is then mapped to a polarization state of a propagating light field (b). Finally, the photonic qubit is mapped back to an atomic state in a separate node by coherently absorbing the photon (c). The results of this single-qubit transfer are reflected in the elements of the process matrix $\chi_{\mathrm{mn}}(\mathbf{d})$. This matrix relates the initial and final density matrices, $\rho_{\mathrm{f}}=\Sigma_{m, n=0}^{3} \chi_{m n} \sigma_{m} \rho_{\mathrm{i}} \sigma_{n}$, where $\sigma_{0}$ is the identity matrix and the other $\sigma_{i}$ are Pauli matrices. Perfect state transfer is achieved when $\chi_{00}=1$. Figure reproduced from ref. 75 , Nature Publishing Group.

a high principal quantum number of $n \approx 100$ (Rydberg state) onto which a photon is mapped ${ }^{46}$, then the strong long-range interaction between two Rydberg atoms can mediate a strong effective photonphoton interaction. In the simplest case of resonant Rydberg EIT (both lasers resonant with their respective transitions, Fig. 4), the strong interaction between two Rydberg atoms tunes the two-photon transition out of resonance, thereby destroying the transparency and leading to absorption ${ }^{22,58,59,62-64}$ for two or more photons. The quantum nonlinearity in this case arises from the Rydberg excitation blockade ${ }^{61}$, which precludes the simultaneous excitation of two Rydberg atoms that are separated by less than the blockade radius $r_{\mathrm{b}}$ (Fig. 4). In essence, such an excited Rydberg system behaves as a 'superatom' consisting of $N_{\mathrm{b}}$ atoms within the blockade radius, with an effective cross-section enhanced by a factor $N_{\mathrm{b}}$, and a corresponding collectively enhanced cooperativity $\eta_{\text {coll }} \approx N_{\mathrm{b}} \lambda^{2} / d^{2}$. Because the blockade prevents multiple excitations, such a superatom behaves as a two-level system, thus resulting in strong optical nonlinearity at the two-photon level when $\eta_{\text {coll }} » 1$.

This idea, first demonstrated in a pioneering experiment by Pritchard and co-workers ${ }^{65,66}$, has recently been used to create a nonlinear optical medium that transmits only one photon, but absorbs two or more photons. As shown by Peyronel and co-workers ${ }^{67}$, such a medium converts incident coherent states of light, consisting of a Poisson distribution in photon number, into outgoing single-photon pulses by absorptive filtering of photon-number states. Related techniques allow the implementation of a deterministic singlephoton source ${ }^{68}$, the switching of light with light ${ }^{69}$, atom-photon entanglement ${ }^{70}$ and the control of light using Rydberg polaritons ${ }^{71}$.

Instead of creating an effective dissipative nonlinearity (that is, a large absorption probability for two photons when they are close to each other), it is also possible to create an effective dispersive nonlinearity - an index of refraction that depends on the distance between the photons. The index of refraction can be viewed as a potential energy because the wavelength in the medium depends on the index of refraction. This implies that two photons can experience an effective distance-dependent potential and force. Firstenberg and co-workers recently implemented such a situation by means of detuned EIT ${ }^{72}$, whereby the control laser is detuned from resonance with the intermediate state, but the two-photon resonance with probe and control laser to the Rydberg state is maintained. In this case, the photons not only travel slowly, but also acquire an effective mass owing to group-velocity dispersion. The attractive interaction between photons in a one-dimensional system then supports a bound state of two photons that manifests itself in the form of photon bunching at the system exit ${ }^{72}$ (Fig. 5). Extensions of this technique should allow for the creation of bound states of three or more photons, which can be viewed as quantum solitons $\mathrm{s}^{73,74}$. 
a

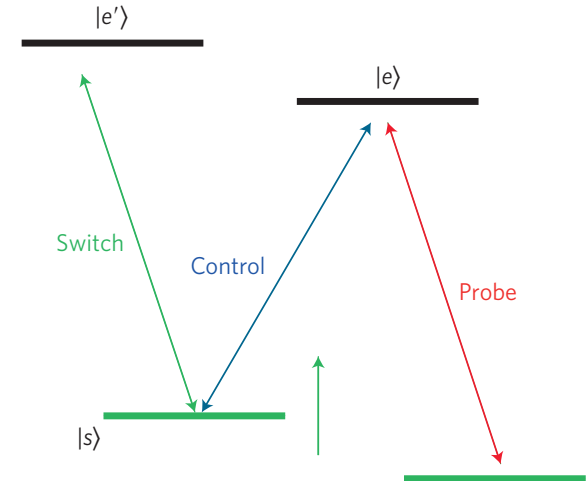

$|g\rangle$
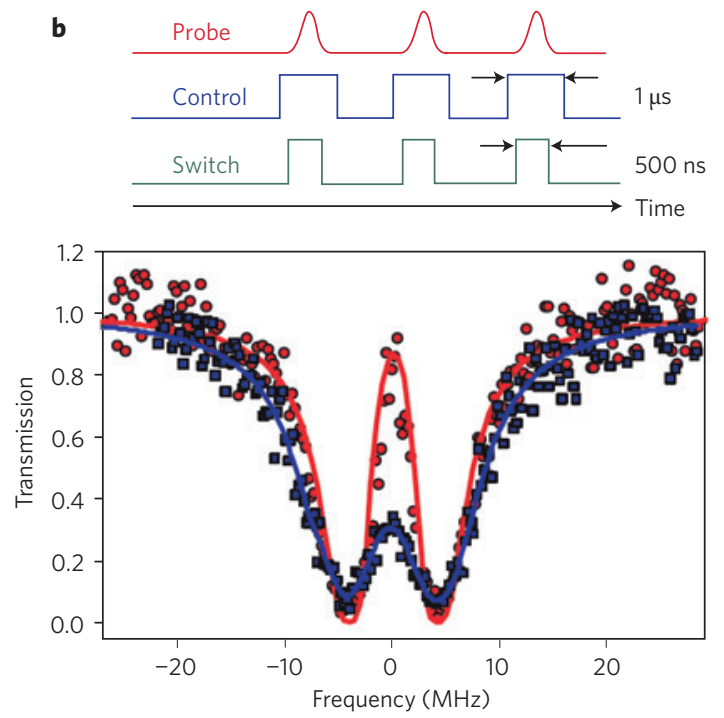

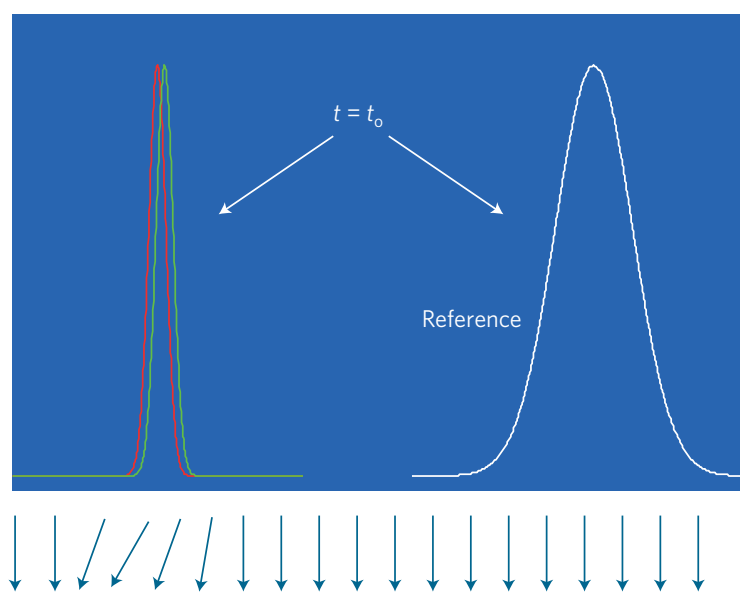

c

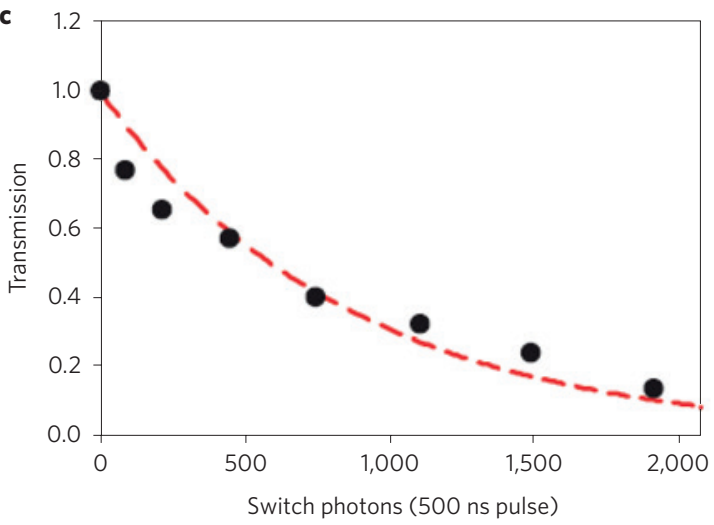

Figure 3 | Nonlinear optics using electromagnetically induced transparency (EIT). a, In EIT, the propagation of a probe field through an otherwise opaque atomic gas is drastically altered by introducing a strong 'control' laser field, which couples the excited state $|e\rangle$ to a metastable state $|s\rangle$. This coupling causes photons in the probe field to mix strongly with spin-wave excitations between states $|g\rangle$ and $|s\rangle$, resulting in a strongly reduced group velocity and the opening of a spectral transparency window for the probe field. The probe field can interact with an additional switch field, which couples state $|s\rangle$ to a second excited state $\left|e^{\prime}\right\rangle$. The switch field destroys the transparency window and causes absorption of the probe field by an amount that depends on the switch intensity. b, Red curve: transmission spectrum for a probe field under standard EIT conditions (that is, without the switch field), which exhibits a narrow transparency window $(T \approx 1)$ around zero probe detuning. Blue curve: the spectrum in the presence of a switching pulse reveals suppression of the transparency. $\mathbf{c}$, Dependence of the probe transmission at zero detuning on the mean number of photons per switching pulse. Parts $\mathbf{b}, \mathbf{c}$, reproduced from ref. 54, (c) 2009 APS.

\section{Applications of quantum nonlinear optics}

Quantum nonlinear systems like those described above constitute a powerful resource for optical quantum information processing. These systems can simultaneously provide both linear operations, such as the storage and state manipulation of photons, and nonlinear operations, such as the generation of single photons on demand and quantum logic between photonic quantum bits. The realization of universal quantum computation requires the implementation of a conditional quantum logic operation (such as a conditional phase shift) between two photons, in combination with single-photon operations (such as polarization rotations). This possibility has served as a major motivation for the development of quantum nonlinear optics ${ }^{6}$. Recent experimental demonstrations within this context include photon-mediated quantum state transfer between atoms in distant cavities ${ }^{75}$, a quantum phase switch between a single atom and a single photon ${ }^{49}$, and a quantum gate between an atom and a photon ${ }^{50}$. A major goal is to scale these systems up to large numbers of qubits, nodes and operations. Such scaling is now becoming possible thanks to the advent of highly efficient atom-photon and photon-photon interactions.
Strongly nonlinear systems also have a number of other important applications. For example, the strong dependence of light propagation on photon number allows the sorting or counting of photons non-destructively ${ }^{48,56,76}$, which, in combination with feedback, could be used to implement various sources of non-classical light fields. Quantum nonlinearities also enable classical nonlinear optical devices, such as routers ${ }^{77,78}$ or all-optical switches, to be operated at their fundamental limit. One example is the case of a singlephoton transistor ${ }^{79}$, in which even a single 'gate' photon can switch hundreds of signal photons $s^{57}$.

Once the generation of single photons and the interactions between individual photons can be reliably controlled, it will be conceptually straightforward to repeat these operations in sequence to generate many-photon (or many-atom) states with a high degree of quantum correlation. In early work, for example, an algorithmic procedure was devised to create an arbitrary photonic state by moving up the nonlinear Jaynes-Cummings energy ladder from the ground state $^{80}$. In a complementary fashion, when several atoms are coupled to a single cavity, strong interactions between the cavity field and 


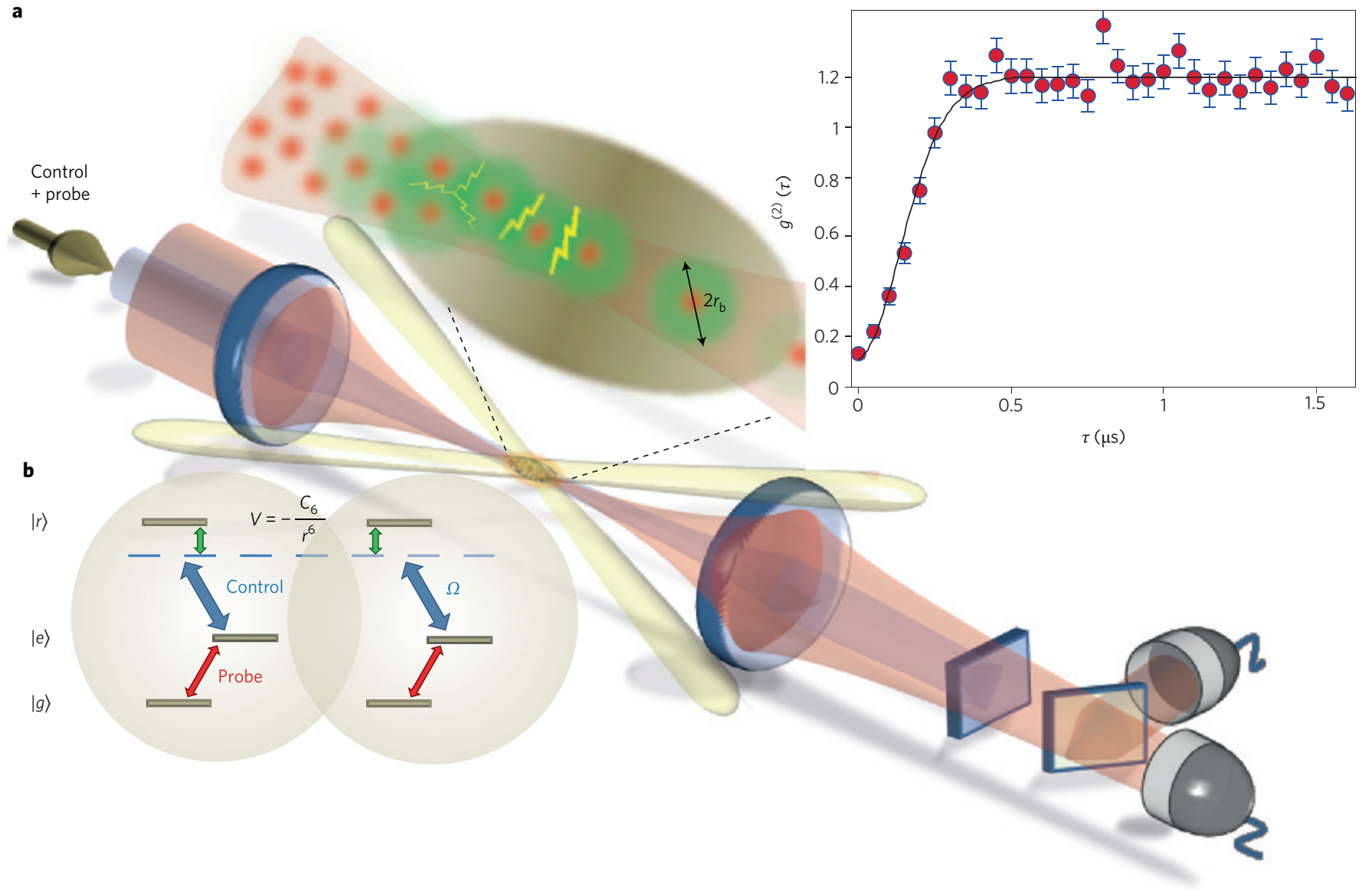

Figure 4 | Rydberg-blockade-mediated interaction between slowly propagating photons. a, Schematic set-up. A strong control beam governs the propagation of a probe beam under electromagnetically induced transparency (EIT) conditions through a cold atomic gas, where a Rydberg state $|r\rangle$ serves as a metastable level. The probe photons, which propagate as polaritons through the medium, strongly interact due to their atomic Rydberg state components. This strong interaction prevents polaritons from propagating when they are closer than the Rydberg 'blockade radius' $r_{b}$, which yields antibunching in the second-order correlation $g^{(2)}(\tau)$ of the transmitted probe field. $\mathbf{b}$, Atomic level scheme used in the Rydberg EIT setup. The probe field couples the atomic state $|g\rangle$ to the short-lived excited state $|e\rangle$, and the control field couples $|e\rangle$ to a Rydberg state $|r\rangle$ of high quantum number $n \approx 100$. An interaction potential $V=-C_{6} / r^{6}$ between two atoms separated by a distance $r$ in their Rydberg states yields an effective nonlinear interaction between polaritons. Here, $C_{6}$ is the atomic van der Waals coefficient. Figure reproduced from ref. 67, Nature Publishing Group.

collective states of the atoms can be exploited to generate many-atom entanglement ${ }^{81}$. Such states could find applications in quantumenhanced metrology ${ }^{8}$ and super-resolution optical imaging ${ }^{7}$.

\section{Many-body physics with strongly interacting photons}

Most proposals based on quantum nonlinear systems envision the production of single photons and controlled interactions between individual photons as sequential building blocks, which are used to generate highly correlated states of light. Instead of building up correlated states in steps, can such complex states emerge from the simultaneous interaction of many photons in a highly nonlinear optical medium? If the answer is yes, then what are the appropriate descriptions of these dynamic, non-equilibrium many-body systems?

The classical limit of this problem has been studied for several decades in nonlinear media, particularly in the context of optical fibres $^{2}$. Here, the nonlinear response of the fibre core, when accumulated over a long propagation distance, can give rise to pronounced effects. For example, it can be used to compensate for group-velocity dispersion through the formation of shape-preserving optical pulses known as solitons, yield propagation instabilities or induce strong mixing with acoustic waves ${ }^{2}$. Achieving a detailed understanding of nonlinear effects in optical fibres has been critical to the advent of high-capacity optical communication networks. A key feature behind the richness of nonlinear phenomena in these optical systems is the continuous spatial (or frequency) degrees of freedom that must be accounted for. (In contrast, the simplest description of cavity QED involves just a single, fixed spatial mode of interest ${ }^{33}$.) Early seminal work towards understanding multimode effects in the quantum domain focused on developing quantum descriptions of solitons ${ }^{73,74}$. This work predicted a number of important effects, such as the possibility of squeezed fluctuations ${ }^{74}$. However, for weak nonlinearities, where the quantum fluctuations associated with a large number of photons are fractionally small, most of the classical properties of solitons are retained in a quantum mechanical description. Complementary work pointed to the possibility of few-photon bound states ${ }^{82-84}$, although the weak nonlinearities associated with fibres made their physical realization unrealistic at the time. Only very recently have such two-photon bound states been observed experimentally in Rydberg EIT ${ }^{72}$ (Fig. 5).

The emergence of physical systems in which these few-photon quantum effects can be experimentally observed has sparked a recent resurgence of interest into the many-body problem of light propagation in strongly nonlinear media. Early proposals envisioned that individual cavities containing quantum emitters could be coupled to form extended arrays ${ }^{85-88}$. The competition between the linear effect of photons hopping between cavities and the interaction of photons occupying the same cavity was predicted to give rise to quantum phase transitions. Other work considered light 

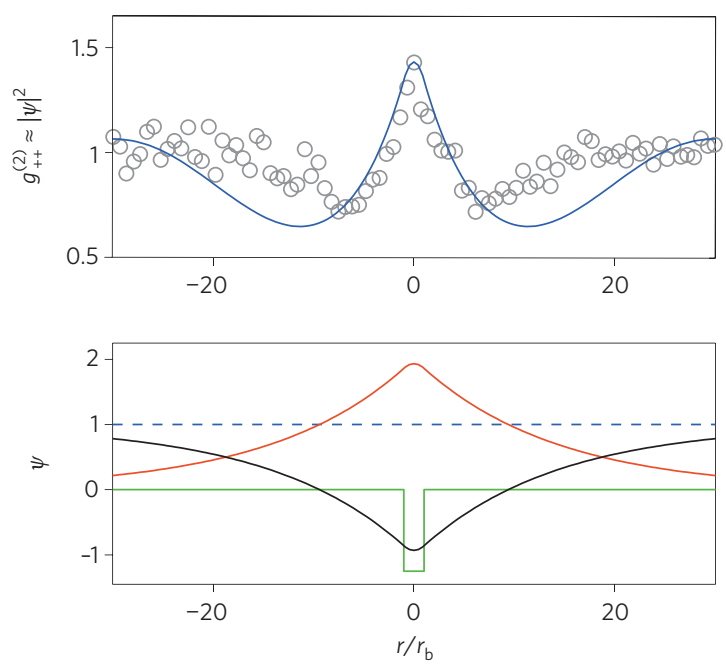

b

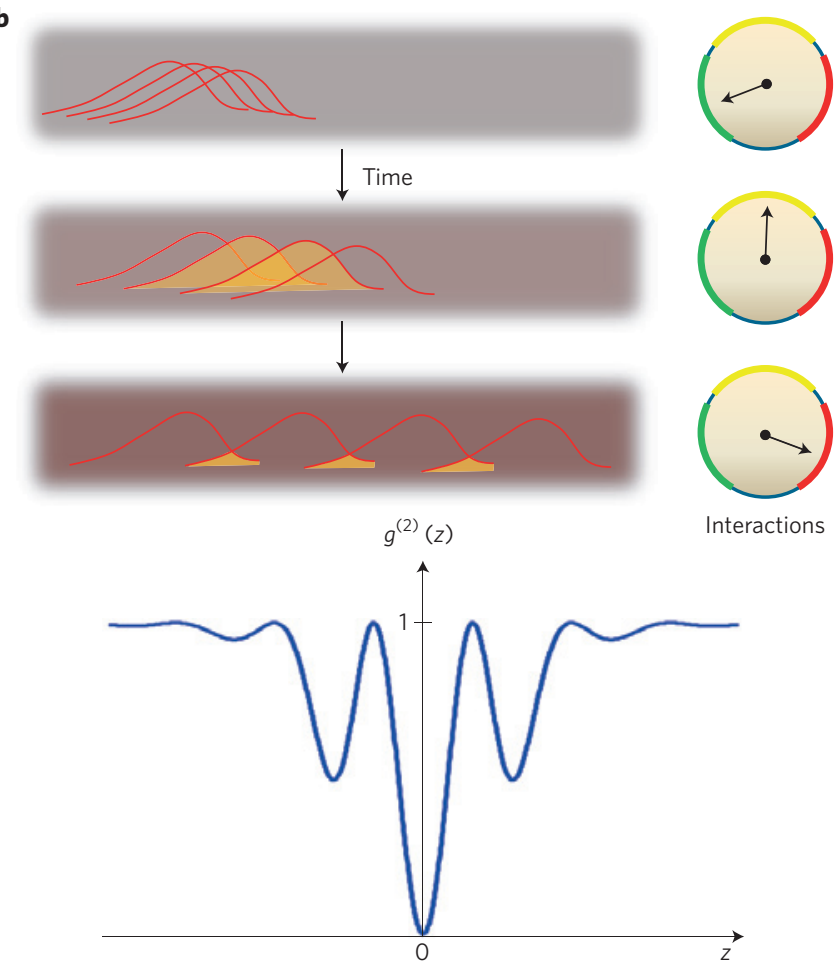

Figure 5 | Many-body quantum dynamics in a nonlinear medium. a, Two-photon dynamics of attractive photons in Rydberg electromagnetically induced transparency (EIT), resulting in a two-photon bound state. Top: photon bunching, observed in the second-order correlation function $g^{(2)}(r)$, which indicates attraction between photons. The cusp-like peak near $r=0$ is a generic feature of a bound state in a one-dimensional system. The circles and blue line indicate experimentally measured values and a theoretical model, respectively. Bottom: The Rydberg EIT medium gives rise to an effective photonphoton interaction potential that can be modelled approximately as a rectangular potential well (green), which supports the two-photon bound-state wavefunction $\psi(r)$ (red). $\mathbf{b}$, In the case of repulsive photons, a strongly nonlinear medium is theoretically predicted to give rise to photon crystallization. Here, a pulse of overlapping photons enters the medium, which is initially tuned to have negligible nonlinear interactions. An increase in repulsive interaction energy (indicated by the shaded regions) causes the optical pulse to spread out and the photons to separate, finally producing a crystal in the limit of very strong interactions. Signatures of crystallization can be observed in second-order correlation measurements, as illustrated in the bottom panel. The correlation function exhibits not only antibunching at $z=0$ (which indicates the absence of overlapping photons) but long-range correlations indicative of crystal-like properties. Part a reproduced from ref. 72, Nature Publishing Group.

propagating in cold atomic gases, in which the photon-photon interactions are induced using either single-atom nonlinearities or strong interactions between atoms in Rydberg states ${ }^{89,90}$. For example, researchers predict that a photonic state exhibiting crystal correlations could be produced by engineering strong effective repulsion between photons and an effective photonic mass in EIT-based systems ${ }^{89,90}$. This represents a remarkable new avenue for quantum nonlinear optics, wherein exotic states of light can be created whose collective properties cannot be understood in a mean-field picture - a type of behaviour that in the past has been associated primarily with strongly interacting condensed-matter systems.

Like their condensed matter counterparts, strongly interacting many-body photonic systems offer a number of challenges and opportunities. For example, effective theories such as Luttinger liquids have proven very useful for describing condensed-matter systems, but it is an open question whether such theories are also applicable to photons ${ }^{90}$. Once we have established a mapping to a model of interest, these strongly interacting photonic systems could be used, for example, to perform quantum simulations of the corresponding Hamiltonian. However, strongly interacting photonic systems have a number of unique aspects when compared with condensed-matter systems: they are intrinsically driven and open - light must be injected and can leak out - and they are generally not coupled to a thermal bath. The nature of phase transitions and photonic correlations in such open, driven systems ${ }^{91}$, and whether dissipation itself can lead to strongly correlated states ${ }^{92}$, will be explored in the coming years. An important task is to develop efficient analytical or numerical techniques for modelling the nonequilibrium quantum dynamics of strongly interacting photons. Recent work in this area focused on applying field-theoretical or condensed-matter techniques, such as S-matrix scattering ${ }^{93-95}$ or density matrix renormalization group ${ }^{92}$.

\section{Outlook}

The rapid development of quantum nonlinear optics in recent years has been driven by the experimental realization of new physical systems that implement strong interactions between atoms and photons. Furthermore, in addition to quantum nonlinearities resulting from single-atom saturation, novel mechanisms and technologies have been identified that could influence the direction of the field.

On the experimental side, significant efforts have been made towards the development of novel nanophotonic elements and integrated interfaces with atoms and solid-state emitters (Fig. 6). For example, the tightly guided modes associated with tapered optical fibres ${ }^{96}$ or photonic crystal waveguides ${ }^{97}$ offer an attractive alternative to free-space focusing. In a recent experiment, Goban and co-workers ${ }^{97}$ demonstrated an interaction probability of $p \approx 0.3$ between a single atom and a single guided photon in a photonic crystal waveguide on a single pass. Ensembles containing more than 1,000 cold atoms have now been coupled to tapered fibres ${ }^{96}$. It is also possible to implement cavity structures with photonic crystals, which enable strong atom-photon interactions by way of 


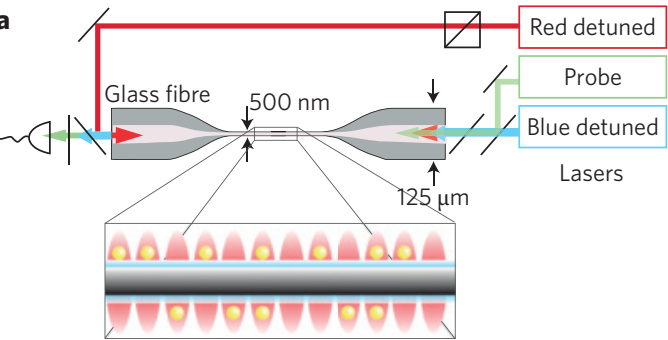

d

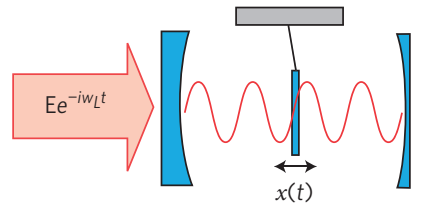

b

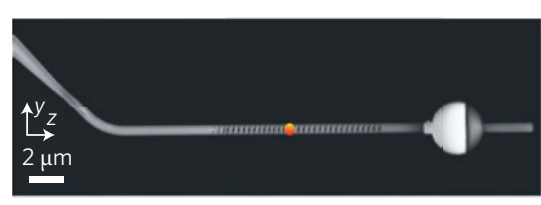

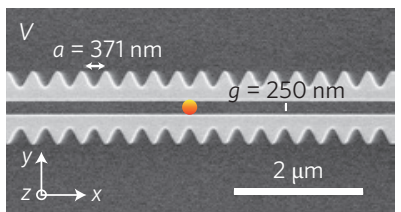

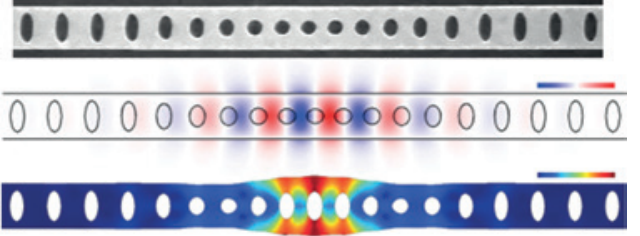

f

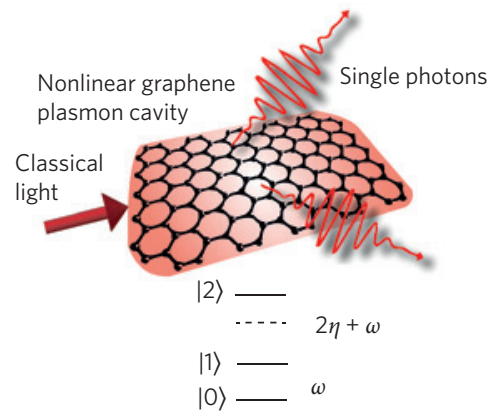

Figure 6 | Quantum interfaces between atoms and nanophotonic systems. a, Simultaneous guided red- and blue-detuned fields through a tapered optical nanofibre can form a one-dimensional optical lattice trap. The strong transverse confinement of the guided modes enables an interaction probability of $p \approx 0.1$ between a single atom and a resonant photon. $\mathbf{b}, \mathrm{A}$ single atom trapped with an optical tweezer near a silicon nitride photonic crystal cavity. To the left is a tapered fibre for coupling light into and out of the structure; to the right is a heating pad for tuning the cavity resonance. c, A single atom (orange dot) coupled to a double-beam silicon nitride photonic crystal waveguide. d, An optomechanical system illustrated as a moving membrane inside a Fabry-Pérot cavity. An increasing optical input intensity exerts an increased radiation pressure force on the membrane, whose motion changes the cavity resonance frequency. The intensity-dependent cavity frequency is analogous to a nonlinear optical Kerr medium. Quantum nonlinear effects can occur when the quantum zero-point displacement of the membrane causes a cavity frequency shift $g_{0}$ that exceeds the cavity linewidth $\kappa$. e, State-of-the-art optomechanical systems based on a photonic crystal device. Simulations depict the optical (top) and mechanical (bottom) resonances. f, Graphene nanostructures can support resonant plasmon modes with mode volumes far below the free-space diffraction limit ( $V_{0}$ " $\lambda_{0}{ }^{3}$ ). In combination with strong nonlocal interactions induced by the free electrons in the material, two plasmons are predicted to interact strongly with one another. Figure reproduced with permission from: a, ref. 96, (c) 2010 APS; b, ref. 49, Nature Publishing Group; c, ref. 97, Nature Publishing Group; d, ref. 103, (c) 2011 APS; e, ref. 104, (c) 2012 AIP; f, ref. 106, (c) 2013 APS.

subdiffraction-limited mode volumes in combination with high cavity quality factors ${ }^{98}$. The ability to interface such nano- and microscopic cavities efficiently with conventional photonic waveguides and fibres ${ }^{77,99}$ makes these systems promising for the practical realization of quantum networks. In addition, remarkable advances in the fabrication of photonic structures from nonconventional optical materials, such as diamond containing nitrogen-vacancy centres $^{39,40}$, may allow for multifunctional quantum devices that simultaneously exhibit strong optical nonlinearities and multiqubit interactions. Strong single-pass interactions between individual emitters and single photons, in the form of surface plasmons focused tightly below the diffraction limit by thin conducting nanowires, have also been recently demonstrated ${ }^{100,101}$.

Furthermore, novel mechanisms for generating strong optical nonlinearities - other than the electronic spectrum of an atom - have recently been identified. For example, optomechanical systems ${ }^{102}$ produce effective optical nonlinearities from motioninduced shifts in the frequency of an optical resonator. It was recently shown that sufficiently large photon-phonon couplings can enable strong interactions between single photons ${ }^{103}$ (Fig. 6d). The state-of-the-art systems are currently within two orders of magnitude of this regime. ${ }^{104}$ (Fig. 6e). This type of nonlinearity might also be explored using atoms as novel light-mass optomechanical elements. Gupta and co-workers have demonstrated that the motion of a Bose-Einstein condensate inside a Fabry-Pérot cavity, in response to the optical potential created by just one intracavity photon, results in a significant alteration of the optical response for subsequent photons ${ }^{105}$. This motion-induced Kerr nonlinearity was sufficiently strong enough to provide optical bistability at the individual-photon level. Finally, there is renewed interest in optical materials that can achieve strong single-photon interactions, as has been proposed recently for graphene ${ }^{106}$ (Fig. 6f). In the wake of recent breakthroughs, the coming years will undoubtedly bring forth exciting advances in the fundamental science of strongly interacting photons, as well as the first practical applications enabled by these techniques.

Received 20 February 2014; accepted 22 July 2014; published online 24 August 2014

\section{References}

1. Boyd, R. W. Nonlinear Optics (Academic, 2003).

2. Agrawal, G. Nonlinear Fiber Optics 5th edn (Academic, 2012).

3. Zipfel, W. R., Williams, R. M. \& Webb, W. W. Nonlinear magic: Multiphoton microscopy in the biosciences. Nature Biotechnol. 21, 1369-1377 (2003).

4. Hell, S. W. Toward fluorescence nanoscopy. Nature Biotechnol. 21, 1347-1355 (2003).

5. Miller, D. A. B. Are optical transistors the logical next step? Nature Photon. 4, 3-5 (2010).

6. Kimble, H. J. The quantum internet. Nature 453, 1023-1030 (2008).

7. Muthukrishnan, A., Scully, M. O. \& Zubairy, M. S. Quantum microscopy using photon correlations. J. Opt. B 6, S575 (2004).

8. Giovannetti, V., Lloyd, S. \& Maccone, L. Advances in quantum metrology. Nature Photon. 5, 222-229 (2011).

9. Franken, P. A. \& Ward, J. F. Optical harmonics and nonlinear phenomena. Rev. Mod. Phys. 35, 23-39 (1963).

10. Byer, R. L. Quasi-phasematched nonlinear interactions and devices. J. Nonlinear Opt. Phys. Mater. 6, 549-592 (1997).

11. Milburn, G. J. Quantum optical Fredkin gate. Phys. Rev. Lett. 62, 2124-2127 (1989).

12. Imoto, N., Haus, H. A. \& Yamamoto, Y. Quantum nondemolition measurement of the photon number via the optical Kerr effect. Phys. Rev. A 32, 2287-2292 (1985).

13. Harris, S. E., Field, J. E. \& Imamoğlu, A. Nonlinear optical processes using electromagnetically induced transparency. Phys. Rev. Lett. 64, 1107-1110 (1990).

14. Turchette, Q. A., Hood, C. J., Lange, W., Mabuchi, H. \& Kimble, H. J. Measurement of conditional phase shifts for quantum logic. Phys. Rev. Lett. 75, 4710-4713 (1995).

15. Haroche, S. \& Raimond, J.-M. Exploring the Quantum: Atoms, Cavities, and Photons (Oxford Univ. Press, 2006). 
16. Devoret, M. H. \& Schoelkopf, R. J. Superconducting circuits for quantum information: An outlook. Science 339, 1169-1174 (2013).

17. Knill, E., Laflamme, R. \& Milburn, G. J. A scheme for efficient quantum computation with linear optics. Nature 409, 46-52 (2001).

18. Pan, J.-W. et al. Multiphoton entanglement and interferometry. Rev. Mod. Phys. 84, 777-838 (2012).

19. Schmidt, H. \& Imamoğlu, A. Giant Kerr nonlinearities obtained by electromagnetically induced transparency. Opt. Lett. 21, 1936-1938 (1996).

20. Harris, S. E. \& Hau, L. V. Nonlinear optics at low light levels. Phys. Rev. Lett. 82, 4611-4614 (1999).

21. Duan, L.-M. \& Kimble, H. J. Scalable photonic quantum computation through cavity-assisted interactions. Phys. Rev. Lett. 92, 127902 (2004).

22. Gorshkov, A. V., Otterbach, J., Fleischhauer, M., Pohl, T. \& Lukin, M. D. Photon-photon interactions via Rydberg blockade. Phys. Rev. Lett. 107, 133602 (2011)

23. Darquie, B. et al. Controlled single-photon emission from a single trapped two-level atom. Science 309, 454-456 (2005).

24. Tey, M. K. et al. Strong interaction between light and a single trapped atom without the need for a cavity. Nature Phys. 4, 924-927 (2008).

25. Hetet, G., Slodicka, L., Hennrich, M. \& Blatt, R. Single atom as a mirror of an optical cavity. Phys. Rev. Lett. 107, 133002 (2011).

26. Wrigge, G., Gerhardt, I., Hwang, J., Zumofen, G. \& Sandoghdar, V. Efficient coupling of photons to a single molecule and the observation of its resonance fluorescence. Nature Phys. 4, 60-66 (2008).

27. Birnbaum, K. M. et al. Photon blockade in an optical cavity with one trapped atom. Nature 436, 87-90 (2005).

28. Schuster, I. et al. Nonlinear spectroscopy of photons bound to one atom. Nature Phys. 4, 382-385 (2008).

29. Fushman, I. et al. Controlled phase shifts with a single quantum dot. Science 320, 769-772 (2008).

30. Dayan, B. et al. A photon turnstile dynamically regulated by one atom. Science 319, 1062-1065 (2008).

31. Volz, T. et al. Ultrafast all-optical switching by single photons. Nature Photon. 6, 605-609 (2012)

32. Tanji-Suzuki, H., Chen, W., Landig, R., Simon, J. \& Vuletic, V. Vacuuminduced transparency. Science 333, 1266-1269 (2011).

33. Jaynes, E. \& Cummings, F. Comparison of quantum and semiclassical radiation theories with application to the beam maser. Proc. IEEE 51, 89-109 (1963).

34. Kubanek, A. et al. Two-photon gateway in one-atom cavity quantum electrodynamics. Phys. Rev. Lett. 101, 203602 (2008).

35. Koch, M. et al. Three-photon correlations in a strongly driven atom-cavity system. Phys. Rev. Lett. 107, 023601 (2011).

36. Michler, P. et al. A quantum dot single-photon turnstile device. Science 290, 2282-2285 (2000)

37. Pelton, M. et al. Efficient source of single photons: A single quantum dot in a micropost microcavity. Phys. Rev. Lett. 89, 233602 (2002).

38. Laucht, A. et al. Electrical control of spontaneous emission and strong coupling for a single quantum dot. New J. Phys. 11, 023034 (2009).

39. Faraon, A., Barclay, P. E., Santori, C., Fu, K.-M. C. \& Beausoleil, R. G. Resonant enhancement of the zero-phonon emission from a colour centre in a diamond cavity. Nature Photon. 5, 301-305 (2011).

40. Hausmann, B. J. M. et al. Integrated diamond networks for quantum nanophotonics. Nano Lett. 12, 1578-1582 (2012).

41. Bajcsy, M. et al. Non-classical three-photon correlations with a quantum dot strongly coupled to a photonic-crystal nanocavity. Preprint at http://lanl.arXiv.org/abs/1307.3601v1 (2013).

42. Reinhard, A. et al. Strongly correlated photons on a chip. Nature Photon. 6, 93-96 (2012).

43. Witthaut, D., Lukin, M. D. \& Sørensen, A. S. Photon sorters and QND detectors using single photon emitters. Europhys. Lett. 97, 50007 (2012).

44. Xu, S., Rephaeli, E. \& Fan, S. Analytic properties of two-photon scattering matrix in integrated quantum systems determined by the cluster decomposition principle. Phys. Rev. Lett. 111, 223602 (2013).

45. Cirac, J. I., Zoller, P., Kimble, H. J. \& Mabuchi, H. Quantum state transfer and entanglement distribution among distant nodes in a quantum network. Phys. Rev. Lett. 78, 3221-3224 (1997).

46. Fleischhauer, M., Imamoğlu, A. \& Marangos, J. P. Electromagnetically induced transparency: Optics in coherent media. Rev. Mod. Phys. 77, 633-673 (2005).

47. Specht, H. P. et al. A single-atom quantum memory. Nature 473, 190-193 (2011).

48. Reiserer, A., Ritter, S. \& Rempe, G. Nondestructive detection of an optical photon. Science 342, 1349-1351 (2013).

49. Tiecke, T. G. et al. Nanophotonic quantum phase switch with a single atom. Nature 508, 241-244 (2014)

50. Reiserer, A. et al. A quantum gate between a flying optical photon and a single trapped atom. Nature 508, 237-240 (2014).
51. Hammerer, K., Sørensen, A. S. \& Polzik, E. S. Quantum interface between light and atomic ensembles. Rev. Mod. Phys. 82, 1041-1093 (2010).

52. Phillips, D. F., Fleischhauer, A., Mair, A., Walsworth, R. L. \& Lukin, M. D. Storage of light in atomic vapor. Phys. Rev. Lett. 86, 783-786 (2001).

53. Liu, C., Dutton, Z., Behroozi, C. H. \& Hau, L. V. Observation of coherent optical information storage in an atomic medium using halted light pulses. Nature 409, 490-493 (2001)

54. Bajcsy, M. et al. Efficient all-optical switching using slow light within a hollow fiber. Phys. Rev. Lett. 102, 203902 (2009).

55. Venkataraman, V., Saha, K., Londero, P. \& Gaeta, A. L. Few-photon all-optical modulation in a photonic band-gap fiber. Phys. Rev. Lett. 107, 193902 (2011).

56. Nikoghosyan, G. \& Fleischhauer, M. Photon-number selective group delay in cavity induced transparency. Phys. Rev. Lett. 105, 013601 (2010).

57. Chen, W. et al. All-optical switch and transistor gated by one stored photon. Science 341, 768-770 (2013).

58. Friedler, I., Petrosyan, D., Fleischhauer, M. \& Kurizki, G. Long-range interactions and entanglement of slow single-photon pulses. Phys. Rev. A 72, 043803 (2005).

59. Moller, D., Madsen, L. B. \& Molmer, K. Quantum gates and multiparticle entanglement by Rydberg excitation blockade and adiabatic passage. Phys. Rev. Lett. 100, 170504 (2008).

60. Rajapakse, R. M. et al. Single-photon nonlinearities using arrays of cold polar molecules. Phys. Rev. A 80, 013810 (2009).

61. Lukin, M. D. et al. Dipole blockade and quantum information processing in mesoscopic atomic ensembles. Phys. Rev. Lett. 87, 037901 (2001).

62. Petrosyan, D., Otterbach, J. \& Fleischhauer, M. Electromagnetically induced transparency with Rydberg atoms. Phys. Rev. Lett. 107, 213601 (2011).

63. Sevincli, S., Henkel, N., Ates, C. \& Pohl, T. Nonlocal nonlinear optics in cold Rydberg gases. Phys. Rev. Lett. 107, 153001 (2011).

64. Muller, M., Lesanovsky, I., Weimer, H., Buchler, H. P. \& Zoller, P. Mesoscopic Rydberg gate based on electromagnetically induced transparency. Phys. Rev. Lett. 102, 170502 (2009).

65. Pritchard, J. D. et al. Cooperative atom-light interaction in a blockaded Rydberg ensemble. Phys. Rev. Lett. 105, 193603 (2010).

66. Pritchard, J., Weatherill, K. \& Adams, C. Nonlinear optics using cold Rydberg atoms in Annual Review of Cold Atoms and Molecules (eds Madison, K. W., Wang, Y., Rey, A. M. \& Bongs, K.) 1, 301-350 (World Scientific, 2013).

67. Peyronel, T. et al. Quantum nonlinear optics with single photons enabled by strongly interacting atoms. Nature 488, 57-60 (2012).

68. Dudin, Y. O. \& Kuzmich, A. Strongly interacting Rydberg excitations of a cold atomic gas. Science 336, 887-889 (2012).

69. Baur, S., Tiarks, D., Rempe, G. \& Dürr, S. Single-photon switch based on Rydberg blockade. Phys. Rev. Lett. 112, 073901 (2014).

70. Li, L., Dudin, Y. O. \& Kuzmich, A. Entanglement between light and an optical atomic excitation. Nature 498, 466-469 (2013).

71. Maxwell, D. et al. Storage and control of optical photons using Rydberg polaritons. Phys. Rev. Lett. 110, 103001 (2013).

72. Firstenberg, O. et al. Attractive photons in a quantum nonlinear medium. Nature 502, 71-75 (2013).

73. Lai, Y. \& Haus, H. A. Quantum theory of solitons in optical fibers. II. Exact solution. Phys. Rev. A 40, 854-866 (1989).

74. Drummond, P. D., Shelby, R. M., Friberg, S. R. \& Yamamoto, Y. Quantum solitons in optical fibres. Nature 365, 307-313 (1993).

75. Ritter, S. et al. An elementary quantum network of single atoms in optical cavities. Nature 484, 195-200 (2012).

76. Grangier, P., Levenson, J. A. \& Poizat, J.-P. Quantum non-demolition measurements in optics. Nature 396, 537-542 (1998).

77. O'Shea, D., Junge, C., Volz, J. \& Rauschenbeutel, A. Fiber-optical switch controlled by a single atom. Phys. Rev. Lett. 111, 193601 (2013).

78. Shomroni, I. et al. All-optical routing of single photons by a one-atom switch controlled by a single photon. Science http://dx.doi.org/10.1126/ science.1254699 (2014).

79. Chang, D. E., Sørensen, A. S., Demler, E. A. \& Lukin, M. D. A single-photon transistor using nanoscale surface plasmons. Nature Phys. 3, 807-812 (2007)

80. Law, C. K. \& Eberly, J. H. Arbitrary control of a quantum electromagnetic field. Phys. Rev. Lett. 76, 1055-1058 (1996).

81. Plenio, M. B., Huelga, S. F., Beige, A. \& Knight, P. L. Cavity-loss-induced generation of entangled atoms. Phys. Rev. A 59, 2468-2475 (1999).

82. Chiao, R. Y., Deutsch, I. H. \& Garrison, J. C. Two-photon bound state in self-focusing media. Phys. Rev. Lett. 67, 1399-1402 (1991).

83. Deutsch, I. H., Chiao, R. Y. \& Garrison, J. C. Two-photon bound states: The diphoton bullet in dispersive self-focusing media. Phys. Rev. A 47, 3330-3336 (1993)

84. Kheruntsyan, K. V. \& Drummond, P. D. Three-dimensional quantum solitons with parametric coupling. Phys. Rev. A 58, 2488-2499 (1998).

85. Hartmann, M. J., Brandao, F. G. S. L. \& Plenio, M. B. Strongly interacting polaritons in coupled arrays of cavities. Nature Phys. 2, 849-855 (2006). 
86. Greentree, A. D., Tahan, C., Cole, J. H. \& Hollenberg, L. C. L. Quantum phase transitions of light. Nature Phys. 2, 856-861 (2006).

87. Angelakis, D. G., Santos, M. F. \& Bose, S. Photon-blockade-induced Mott transitions and spin models in coupled cavity arrays. Phys. Rev. A 76, 031805 (2007).

88. Carusotto, I. \& Ciuti, C. Quantum fluids of light. Rev. Mod. Phys. 85, 299-366 (2013).

89. Chang, D. E. et al. Crystallization of strongly interacting photons in a nonlinear optical fibre. Nature Phys. 4, 884-889 (2008).

90. Otterbach, J., Moos, M., Muth, D. \& Fleischhauer, M. Wigner crystallization of single photons in cold Rydberg ensembles. Phys. Rev. Lett. 111, 113001 (2013).

91. Carusotto, I. et al. Fermionized photons in an array of driven dissipative nonlinear cavities. Phys. Rev. Lett. 103, 033601 (2009).

92. Kiffner, M. \& Hartmann, M. J. Dissipation-induced correlations in onedimensional bosonic systems. New J. Phys. 13, 053027 (2011).

93. Shen, J.-T. \& Fan, S. Strongly correlated two-photon transport in a one-dimensional waveguide coupled to a two-level system. Phys. Rev. Lett. 98, 153003 (2007).

94. Shi, T. \& Sun, C. P. Lehmann-Symanzik-Zimmermann reduction approach to multiphoton scattering in coupled-resonator arrays. Phys. Rev. B 79, 205111 (2009).

95. Pletyukhov, M. \& Gritsev, V. Scattering of massless particles in onedimensional chiral channel. New J. Phys. 14, 095028 (2012).

96. Vetsch, E. et al. Optical interface created by laser-cooled atoms trapped in the evanescent field surrounding an optical nanofiber. Phys. Rev. Lett. 104, 203603 (2010).

97. Goban, A. et al. Atom-light interactions in photonic crystals. Nature Commun. 5, 3808 (2014)

98. Thompson, J. D. et al. Coupling a single trapped atom to a nanoscale optical cavity. Science 340, 1202-1205 (2013)

99. Volz, J., Scheucher, M., Junge, C. \& Rauschenbeutel, A. Nonlinear phase shift for single fiber-guided photons interacting with a single atom. Preprint at http://lanl.arXiv.org/abs/1403.1860v1 (2014).

100. Akimov, A. V. et al. Generation of single optical plasmons in metallic nanowires coupled to quantum dots. Nature 450, 402-406 (2007).

101. Kolesov, R. et al. Wave-particle duality of single surface plasmon polaritons. Nature Phys. 5, 470-474 (2009).
102. Aspelmeyer, M., Groblacher, S., Hammerer, K. \& Kiesel, N. Quantum optomechanics - throwing a glance. J. Opt. Soc. Am. B 27, A189-A197 (2010)

103. Rabl, P. Photon blockade effect in optomechanical systems. Phys. Rev. Lett. 107, 063601 (2011).

104. Chan, J., Safavi-Naeini, A. H., Hill, J. T., Meenehan, S. \& Painter, O. Optimized optomechanical crystal cavity with acoustic radiation shield. Appl. Phys. Lett. 101, 081115 (2012).

105. Gupta, S., Moore, K. L., Murch, K. W. \& Stamper-Kurn, D. M. Cavity nonlinear optics at low photon numbers from collective atomic motion. Phys. Rev. Lett. 99, 213601 (2007).

106. Gullans, M., Chang, D. E., Koppens, F. H. L., de Abajo, F. J. G. \& Lukin, M. D. Single-photon nonlinear optics with graphene plasmons. Phys. Rev. Lett.

111, 247401 (2013).

\section{Acknowledgements}

The authors acknowledge C. Adams, I. Cirac, W. Chen, Y. Chu, E. Demler, M. Fleischhauer, O. Firstenberg, S. Harris, V. Gritsev, A. Gorshkov, A. Imamoğlu, H. J. Kimble, N. de Leon, M. Loncar, H. Tanji-Suzuki, T. Peyronel, T. Pohl, G. Rempe, A. Sørensen, M. O. Scully, T. Tiecke, J. Thompson, J. Vuckovic, S. Yelin, A. Zibrov, and P. Zoller for the many discussions and contributions that resulted in the work described in this Review. Support from the National Science Foundation, Harvard-MIT Center for Ultracold Atoms, Defense Advanced Research Projects Agency, Air Force Office of Scientific Research Multidisciplinary University Research Initiative, Packard Foundation, and European project Graphene-Based Single-Photon Nonlinear Optical Devices (GRASP) is gratefully acknowledged. D.E.C. acknowledges support from Fundacio Privada Cellex Barcelona and the Ramon y Cajal programme.

\section{Author contributions}

The authors contributed equally to this work.

\section{Additional information}

Reprints and permissions information is available online at www.nature.com/reprints. Correspondence and requests for materials should be addressed to M.D.L.

\section{Competing financial interests}

The authors declare no competing financial interests. 